\title{
Wind Turbine Bearing Fault Detection Using Adaptive Resampling and Order Tracking
}

\author{
Cody Walker ${ }^{1}$ and Jamie Coble ${ }^{2}$ \\ ${ }^{1,2}$ University of Tennessee Knoxville, Knoxville, TN, 37996, US \\ cwalke42@utk.edu \\ jamie@utk.edu
}

\begin{abstract}
Wind energy is growing increasingly popular in the United States, so it is imperative to make it as cost competitive as possible. Operations and Maintenance (O\&M) make up $20-25 \%$ of the total cost of onshore wind projects. Unplanned maintenance contributes approximately $75 \%$ of the total maintenance costs (WWEA, 2012). Condition-based maintenance strategies intend to maximize the uptime by reducing to the amounts of unplanned maintenance. This should result in an overall decrease in the cost of maintenance. Wind turbines produce an interesting challenge, because their main shaft rotation is both slow and nonstationary. Through the use of adaptive resampling and order tracking, both of these challenges were combated as the bearing fault was identified in the order spectrum then tracked as it progressed. The fault was identified as an outer race defect on the main bearing that initiated sometime during or before installation. The total energy in the order spectrum around the bearing fault rate was identified as a potential front-runner for a prognostic parameter. This paper presents a case study application to operational wind turbine bearing data to demonstrate the ease and intuitiveness of combining adaptive resampling and order tracking to diagnose faults for slow, nonstationary bearings. Prognosis of remaining useful life is proposed with features extracted from the order spectrum, but additional data are needed to develop and demonstrate this analysis.
\end{abstract}

\section{INTRODUCTION}

Bearings are an essential part of most power transmission devices. Bearings are associated with spinning devices, such as turbines, generators, shafts, and motors. If a bearing fails, that device is no longer able to complete its task. Bearings are notably the leading cause of failure in rotating equipment (Williams, Ribadeneira, Billington, \& Kurfess, 2001).

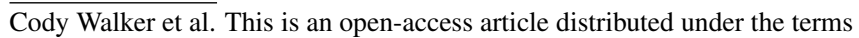
of the Creative Commons Attribution 3.0 United States License, which permits unrestricted use, distribution, and reproduction in any medium, provided the original author and source are credited.
}

In many cases, this can lead to an unplanned outage as the turbine must stop all related activities to fix the broken piece of equipment. This takes away from production time and can be very costly. If we were able to predict when a certain component will fail, we would be able to mitigate the negative effects of this outcome by changing to a more optimized operating condition or by replacing or maintaining the component during a scheduled maintenance outage before it breaks. This so-called condition-based maintenance (CBM) specifically for wind turbine applications has been well-studied in the recent literature; CBM systems rely on early fault detection and component or system health assessment. Three types of signals being explored include: vibration, acoustic emission, and oil analysis. Vibration is one of the most common signals analyzed in the wind turbine industry. Vibration analysis can be used for online monitoring and fault detection with the signals being improved through filtering techniques to enhance the signal-to-noise ratio. Multidimensional features from the signal can then be collapsed into a single feature and used for fault classification (Li \& Frogley, 2013). Vibration analysis has also been used for predicting wind turbine gearbox health using techniques such as adaptive neuro-fuzzy inference system and nonlinear autoregressive model with exogenous inputs (Hussain \& Gabbar, 2013). Acoustic emission (AE) analysis has been applied to diagnosis the health of structural components, but can also be used to defects and imbalances in rotating machinery (Niknam, Thomas, Hines, \& Sawhney, 2013). Oil analysis can provide extremely early warnings for impending machine malfunctions. Online monitoring using particle filtering and measurements of the oil's viscosity and dielectric constant can be used as performance parameters for system health and prediction of the system's remaining useful life (Zhu, Yoon, He, Qu, \& Bechhoefer, 2013).

Bearings can take on a variety of different forms, ranging from ball or roller bearings to more exotic types such as planetary bearings. The basic design of a bearing consists of a rolling element, a cage, an inner race, and an outer race; these components are shown for a standard ball bearing in Figure 


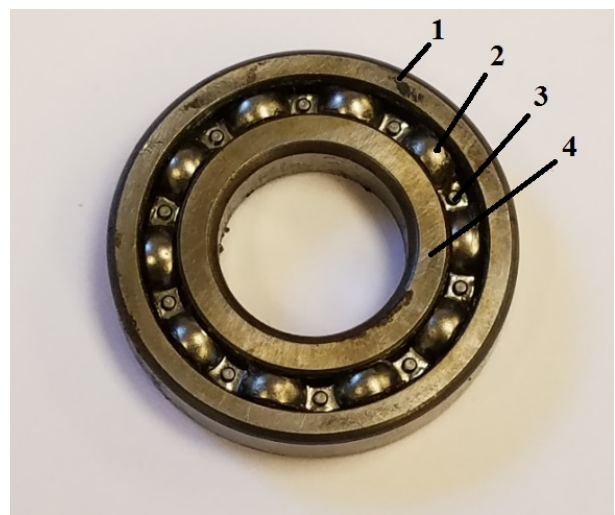

Figure 1. A standard ball bearing with major components: 1. Outer race, 2. Ball, 3. Cage, 4. Inner race.

1. This system is lubricated, typically with oil, to prevent undesired friction between the metal elements. When a fault occurs, vibration analysis can be used to diagnose the defective component. As the bearing spins, the rolling elements will pass by the defect creating an impact much like a car driving over a pothole. The impact frequency is different for each element of the bearing; each element has its own characteristic defect frequency (Rai \& Mohanty, 2006). Frequency analysis for rotating components is typically done through a Fourier transform. This approach, however, is not appropriate for wind turbines whose rotational speed is nonstationary.

By implementing on bearing fault detection and diagnostics and failure prognostics, we can develop a CBM plan to reduce the number and duration of unplanned outages and subsequent costs. CBM is a four step process: data collection, data monitoring, fault diagnostics, and prognostics (Jardine, Lin, \& Banjevic, 2006). The current work focuses on data monitoring and fault diagnostics for drivetrain bearings in wind turbines. The analysis proposed for fault detection and diagnosis may be extensible to support prognostics as well, though more data are needed to evaluate the efficacy of this approach. Prognostics is the ultimate enabling technology for CBM. The estimated remaining useful life is used to make informed decisions concerning the component's operating conditions and future maintenance schedules.

This paper presents a case study application of adaptive resampling and order tracking to address limitations of traditional frequency analysis for nonstationary wind turbine bearings. Data from three wind turbine drivetrains are investigated; these data include two healthy drivetrains and one faulted drivetrain. The following section provides a brief background on bearing fault detection and identification, frequency analysis, and order tracking. Section 3 presents the adaptive resampling and order tracking algorithms proposed. The wind turbine bearing data are described in Section 4 followed the results of the fault detection and diagnosis in Section 5. Potential health indicators are suggested for bearing prognosis, though sufficient data are not available to demonstrate these indicators. Finally conclusions and areas of potential future development are given in Section 6.

\section{BACKGROUND}

Some instigators of bearing faults include manufacturing error, improper installation, inadequate lubrication, and lubricate contamination (Tandon \& Choudhury, 1999). During installation, proper handling procedures must be followed. A clean environment is ideal. Even the smallest contaminate can lead to three body abrasion, bearing wear, and premature failure. Improper installation can also directly damage the bearing elements. Unintended forces, such as a hammer blow, can dent the outer ring, rupture the inner ring, or press the rolling elements into the races. An indention on either raceway, called brinelling, leads to significant damage. Each rolling element traverses the indention at each rotation, which can exacerbate the problem with each pass. Lubrication is essential for the longevity of the bearing. Under-filling, overfilling, water contamination, particulate contamination, lubrication mixing, and over-heating all can cause lubrication failure in bearings. Lubrication failure leads to an inadequate barrier between the rolling elements and the races. Excessive metal-to-metal contact leads to overheating and eventually bearing failure. Lastly, overloading a bearing is simply putting the bearing in a situation that it was not designed to withstand. These design limits include temperature, load, and shaft speed. Exceeding any of these limits can shorten the bearing's life.

Defects on any of the main elements of the bearing lead to characteristic defect frequencies in the frequency domain. The characteristic defect frequency is a function of bearing geometry and shaft frequency. As the shaft spins faster, the bearing's characteristic defect frequencies increase; because the number of impacts in a given time period increases. The equations used to calculate a ball bearing's characteristic defect frequency for the ball spin, outer race frequency, inner race frequency and fundamental train frequency are given in equations $1-4$, respectively.

$$
\begin{gathered}
f_{b s}=f_{s} \frac{p_{d}}{b_{d}}\left(1-\frac{b_{d}^{2}}{p_{d}^{2}} \cos ^{2} \beta\right) \\
f_{o r}=f_{s} \frac{N_{b}}{2}\left(1-\frac{b_{d}}{p_{d}} \cos \beta\right) \\
f_{i r}=f_{s} \frac{N_{b}}{2}\left(1+\frac{b_{d}}{p_{d}} \cos \beta\right) \\
f_{f t}=\frac{f_{s}}{2}\left(1-\frac{b_{d}}{p_{d}} \cos \beta\right)
\end{gathered}
$$




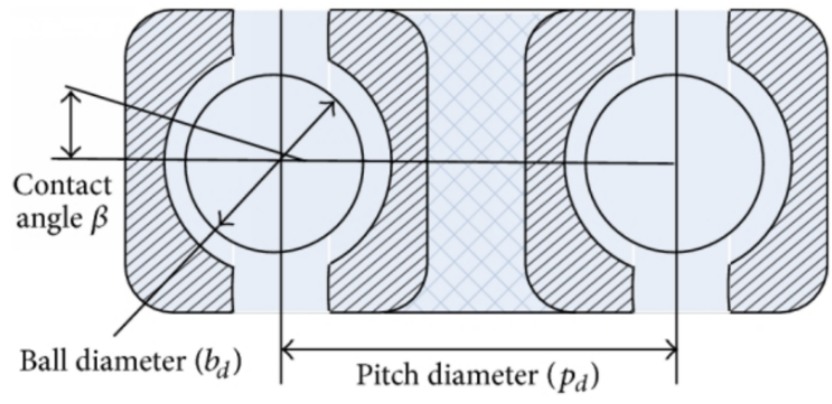

Figure 2. Primary dimensions of a ball bearing (Zhang et al., 2014).

where $\mathrm{b}_{d}$ is the ball diameter, $\mathrm{p}_{d}$ is the pitch diameter, $\mathrm{N}_{b}$ is the number of balls, $\beta$ is the contact angle, $\mathrm{f}_{s}$ is the frequency of the shaft, $\mathrm{f}_{b s}$ is the ball spin frequency, $\mathrm{f}_{o r}$ is the outer race frequency, $\mathrm{f}_{i r}$ is the inner race frequency, and $\mathrm{f}_{f t}$ is the fundamental train frequency, also known as the cage pass frequency (Rai \& Mohanty, 2006). The fundamental train frequency is used to locate faults in the bearing cage. Figure 2 shows the dimensions of a bearing through a schematic of the bearing's cross section.

When analyzing a vibration signal, it is commonplace to use a Fast Fourier Transform (FFT). The FFT transforms the timedomain signal into the frequency domain through the use of complex exponentials (Rai \& Mohanty, 2006). It is much easier to see trends in periodic data through measurement of the frequency content. The FFT yields the signal's average frequency content over the time period. By its definition, the FFT assumes that the signal is stationary (Colombo, Arora, Depace, \& Vinik, 2015). As mentioned previously, the signal is nonstationary due to changes in the shaft's frequency. It would be inappropriate to use the FFT directly. Mutliple methods can be implemented to analyze this type of non-stationary data including: the Hilbert transform (Huang \& Shen, 2014), time synchronous averaging (Bechhoefer \& Kingsley, 2009) and adaptive resampling (Blough, 2006).

The Hilbert transform preserves the signal's shape, amplitude, and phase relations by decomposing the nonstationary signal into elementary time-varying components. The Hilbert transformation is not a transformation into a new domain, but more closely resembles a linear filter. This technique allows the energy of each instantaneous frequency to be calculated. Although the Hilbert transform has proven useful in some applications, there are still doubts regarding the Hilbert transform's reliability as counterexamples and paradoxes have been found (Feldman, 2011).

Time synchronous averaging is a technique used to minimize or eliminate frequencies that are not synchronized with the shaft frequency. Time synchronous averaging takes each rotation of the shaft and averages those signals in the time domain. This has proved to be an effective technique for gears on a nonstationary shaft, because the gears experience no slippage. Their rotational speed is in-sync with the shaft. This technique is less effective for bearings, because they're quasistationary. As the bearings turn, they can experience some slippage, so the bearing's rates are not perfectly in-sync with the shaft. By using time synchronous averaging, the bearing's signal is effectively averaged out (Bechhoefer, Hecke, \& He, 2013). Another technique, such as adaptive resampling, must be used to properly capture the bearing's signal. Adaptive resampling has proven to be an effective technique in analyzing nonstationary, bearing vibration signals.

\section{AdAptive ReSAMPLing}

The goal of adaptive resampling is to convert the original dataset that contains time-varying frequency components into a dataset that appears to be time invariant. After this process, techniques that rely on a stationary dataset can be used. Fourier transform and wavelets are two examples of such techniques (Blough, 2006).

An example of a stationary, time invariant signal can be seen in Figure 3. This figure consists of four plots. The top-most plot shows the frequency over time, which is constant for the first example. The second plot marks the relative times at which the data is sampled. Sensors typically have a constant sampling rate, so the time space between each sample should be consistent. The third plot marks where on the shaft's axis the sample is being taken with an ' $X$ '. From the third plot, we can determine the sample spacing relative to the shaft's angle, $\theta$. This plot shows a constant 8 samples/revolution. The final plot shows the sampled waveform in the angle domain. For the case of a stationary signal, sampling with the respect to the time domain or angle domain does not make a difference. The constant $\Delta \mathrm{t}$ in the time domain leads to a constant $\Delta \theta$ in the angle domain (Blough, 2006). An FFT can be applied to the sampled waveform with no concern.

Figure 4 shows a similar series of plots as Figure 3 with the exception that the frequency is increasing as a function of time. Again, the sampling $\Delta \mathrm{t}$ in the time domain is held constant. As the shaft frequency increases, the angular position of each sample changes. A constant $\Delta \mathrm{t}$ in the time domain no longer corresponds to a constant $\Delta \theta$ in the angle domain. The final plot in Figure 4 shows the sampled waveform in the angle domain. The frequency of the waveform is slowly increasing, so naturally this waveform will not reside in one spectral line in the frequency spectrum (Blough, 2006). This smearing becomes problematic, because the shaft's frequency is used in many processing applications. A smeared shaft frequency will lead to smeared characteristic defect frequency making the fault harder to detect.

Figure 5 has the same steadily increasing function as Figure 4. In this case however, adaptive resampling is used to ensure a constant angular spacing. A variable $\Delta \mathrm{t}$ in the time domain 

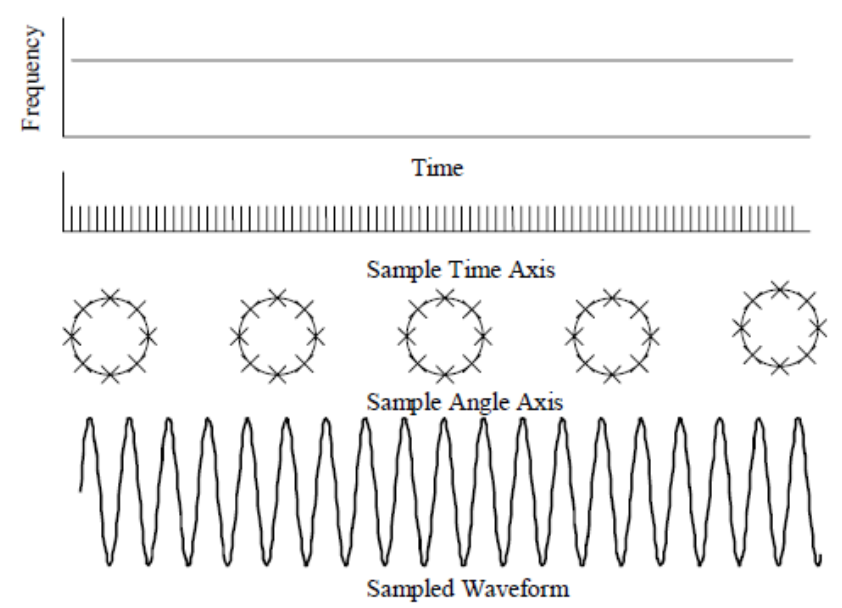

Figure 3. Sampling a stationary signal with constant $\Delta \mathrm{t}$ in the time domain leads to a constant $\Delta \theta$ in the angular domain (Blough, 2006).
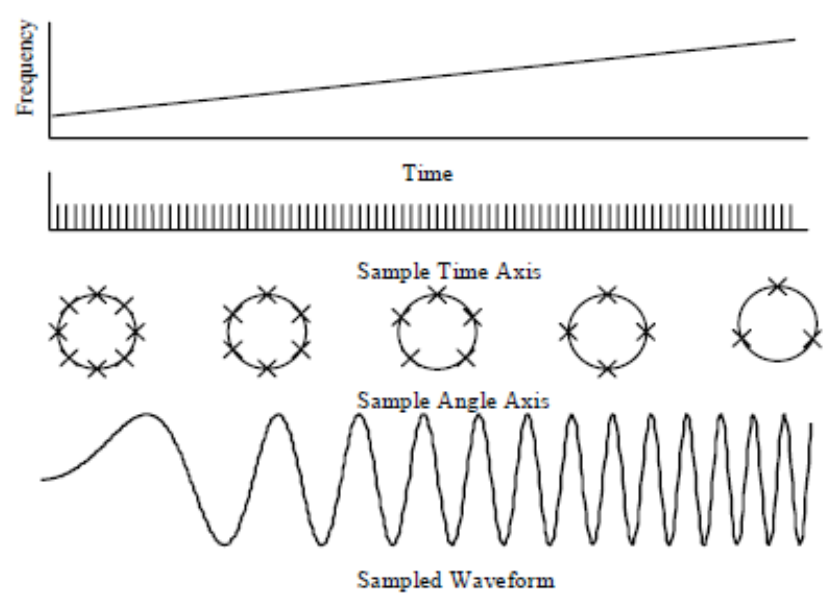

Figure 4. Sampling a non-stationary signal with constant $\Delta \mathrm{t}$ in the time domain leads to a non-constant $\Delta \theta$ in the angular domain (Blough, 2006).

leads to a constant $\Delta \theta$ in the angular domain. This is done in practice through the use of a tachometer (Blough, 2006). The tachometer provides a reference signal, so the time domain can be correlated to the angle domain. As the frequency of the shaft increases or decreases, the number of samples taken in the time domain must also be increased or decreased accordingly. The newly sampled waveform is now based in the angle domain, which may be referred to as the order domain. The varying frequency component that is originally used to align the angle domain is the reference frequency. This frequency is said to be the 1 st order. For the wind turbine system, the 1 st order is the shaft frequency. Equations 1-4 show the characteristic defect frequencies for various faults commonly found in a bearing. All of these frequencies have a linear relationship with the shaft frequency. With the shaft order being one, the bearing characteristic fault rates of $0.45,10.8,12.7$, and

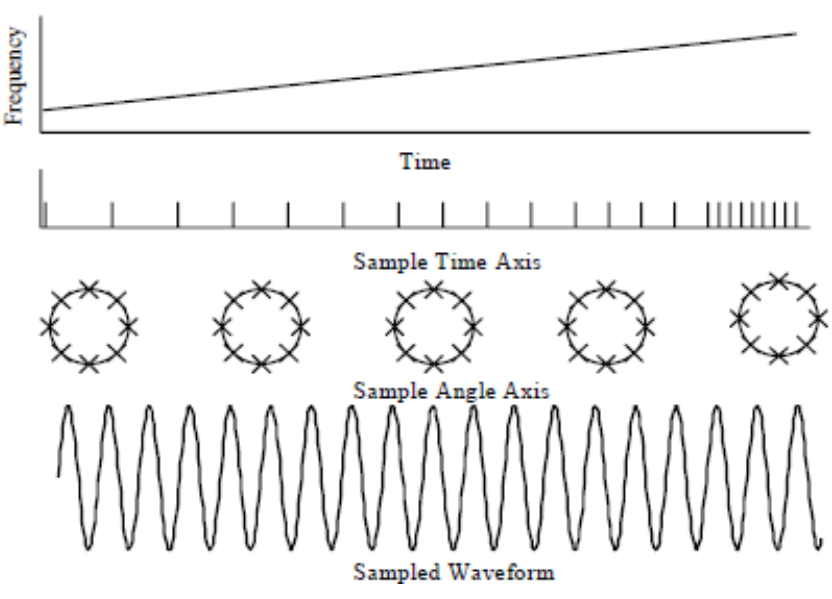

Figure 5. Sampling a non-stationary signal with a resampled (non-constant) $\Delta \mathrm{t}$ in the time domain leads to a constant $\Delta \theta$ in the angular domain (Blough, 2006).

15.3 are the orders of those faults, respectively. The magnitude of the fault's order in the order domain can then be used to diagnose which fault is present in the system.

As the waveform has been converted from the time domain to the angle domain, so must the functions used to analyze them. The formula for the generalized Fourier series in the time domain is given by equation 5 (Tolstov, 1962):

$$
f(x)=a_{0}+\sum_{n=1}^{\infty} a_{n} \cos (n t)+\sum_{n=1}^{\infty} b_{n} \sin (n t)
$$

where the coefficients are

$$
\begin{gathered}
a_{n}=\frac{1}{\pi} \int_{-\pi}^{\pi} f(t) \cos (n t) d t \\
b_{n}=\frac{1}{\pi} \int_{-\pi}^{\pi} f(t) \sin (n t) d t \\
a_{0}=\frac{1}{2 \pi} \int_{-\pi}^{\pi} f(t) d t
\end{gathered}
$$

The analogous coefficients for the Fourier series in the angular domain can be seen in equations 9 and 10 .

$$
\begin{aligned}
& a_{m}=\sum_{n=1}^{N} f(n \Delta \theta) \cos \left(2 \pi o_{m} n \Delta \theta\right) \\
& b_{m}=\sum_{n=1}^{N} f(n \Delta \theta) \sin \left(2 \pi o_{m} n \Delta \theta\right)
\end{aligned}
$$




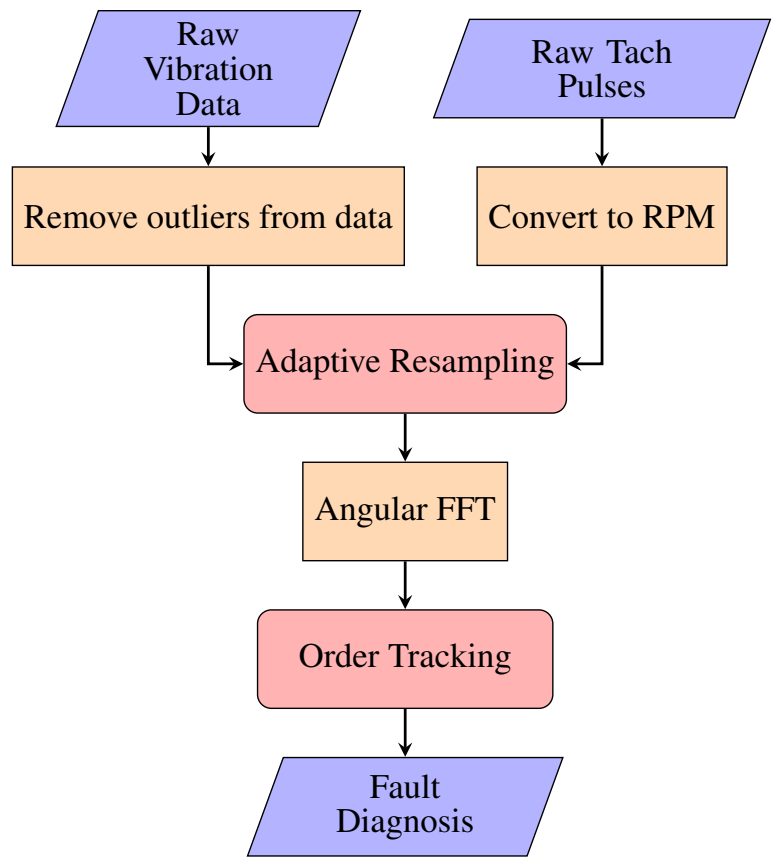

Figure 6. Analysis algorithm to combine adaptive resampling and order tracking for fault detection and diagnosis.

$$
\Delta \theta=\frac{1}{N * \Delta \text { theta }}
$$

where $\mathrm{N}$ is the total number of sample points, $\Delta \theta$ is the angular spacing between the resampled samples and $\mathrm{o}_{m}$ is the order being analyzed. Equation 11 shows the resulting order spacing for the order spectrum as a function of total sample points and size of angular spacing (Blough, 2006).

Figure 6 summaries the steps taken to implement adaptive resampling and order tracking. The initial, raw vibration data is first cleaned by removing any erroneous data entries or obvious outliers. The tach pulses are used to calculate the RPM of the shaft at any given moment. Adaptive resampling uses both the vibration data and the shaft velocity to convert the nonstationary, time-domain signal into a stationary, angular domain signal. An FFT in the angular domain extracts the frequencies from the signal. Order tracking then allows us to easily locate and determine if any faults are present.

\section{Wind Turbine Bearing Data}

The bearing data sets to be analyzed are from the main rotor and carrier bearings of three separate wind turbines. Relative locations of these bearings inside a wind turbine fuselage are shown in Figure 7. We focus primarily on the bearings' vibrations attained from accelerometers placed close to the bearings for fault detection. The accelerometers have a sampling rate of 3,052 samples/second over a 120 second acquisition period. This gives each of the data sets 366,240 points of

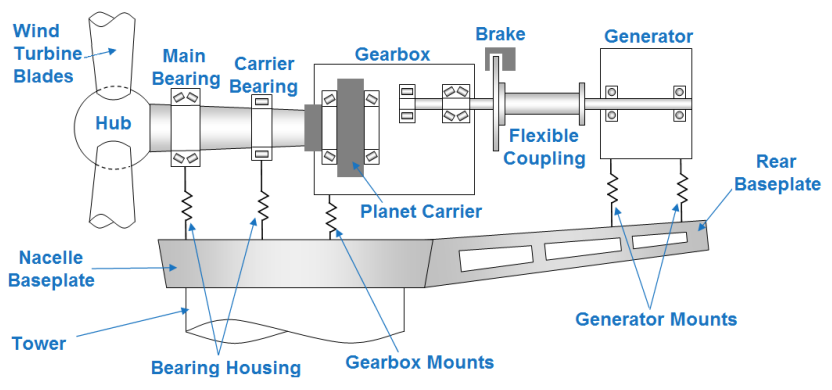

Figure 7. Schematic of a wind turbine two-point mounted high-speed drivetrain (Zipp, 2012). The hub holds the wind turbine's blades. The main and the carrier bearings are located in front (to the left in the figure) of the gearbox, and are typically spherical roller bearings. The main bearing is the primary focus of this paper.

data per acquisition. Measurements of the main shaft's speed were taken simultaneously with a tachometer.

The dimensions of each bearing are unknown, but the rates of characteristic defect frequencies are given. The rate is a dimensionless ratio between the fault frequency and the shaft frequency. The fault frequency will change as the shaft frequency changes, as seen in equations $1-4$. The rate is useful, because it is constant. The cage, ball, outer race, and inner race rates are $0.45,10.8,12.7$, and 15.3 , respectively. By knowing the shaft frequency, we can use these rates to determine characteristic defect frequencies. This task is trivial when the shaft frequency is stationary, or constant, but this is not the case for a wind turbine shaft. Variations as large as 5\% in two seconds can be seen in the shaft's speed. This can be due to: bandwidth limits in the control system, varying loads from the generator, shifting wind conditions, torque ripple, or tower shadow (Dolan \& Lehn, 2006). The control system cannot be expected to keep the shaft at a perfectly constant speed, but rather at a speed within a prescribed bandwidth. When the speed is outside of this bandwidth, control actions are taken to correct the speed. Variations in the electrical load of the generator will produce in a change in the mechanical load that the generator shaft sees. The change in load can be seen in the change in shaft speed. Wind speeds are time varying due to gusts and other common weather conditions. Torque ripple is a change in the torque seen by the rotor as the magnetic fields of the stator and rotor repel one another as the rotor spins. This could be due to the flux density distribution around the airgap or deficiencies in the wiring geometry (Holtz \& Springob, 1996). Tower shadow occurs when the wind turbine blades pass in front of the wind turbine tower. The lift that drives the blades to spin is reduced, because the wind is stalled in front of the tower (Dolan \& Lehn, 2006). All of these effects contribute to the nonstationary nature of the shaft frequency. 


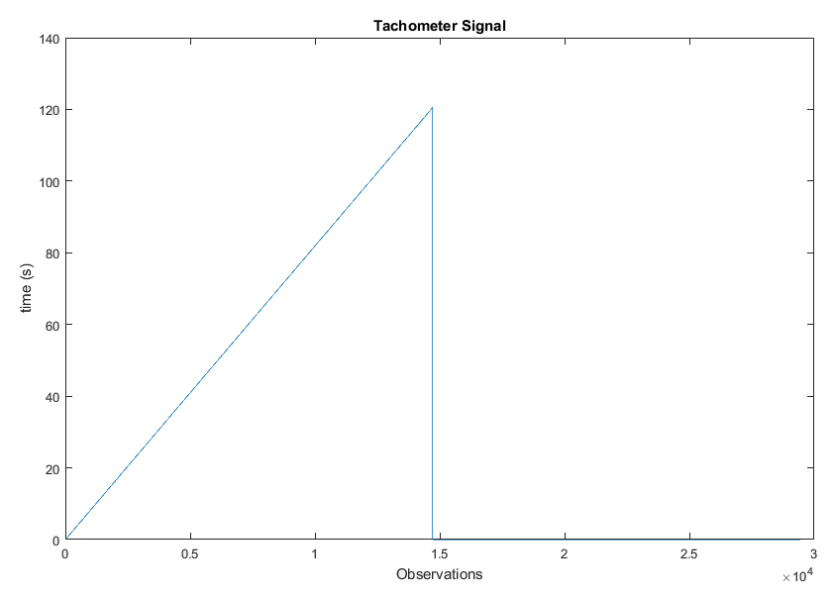

Figure 8. Measured tachometer signal during 120 second acquisition period.

\section{RESUltS}

The main and carrier bearing in three different wind turbine systems were monitored with accelerometers. These systems are slow with non-stationary rotational velocities. Adaptive resampling and order tracking were used to diagnose and track potential faults.

\subsection{Data Resampling}

Adaptive resampling consists of three general steps: obtaining a reference signal, resampling from time to angle domain, and performing an angle domain FFT. The tachometer signal in Figure 8 is the reference signal, which is used to acquire time points of equal angular intervals. The tachometer signal was given in terms time versus tach pulse. Although this is unintuitive, it can still be useful.

The value of tach pulse/second can be calculated by taking the difference between adjacent points from Figure 8. Equation 12 can then be used to calculate the RPM of the shaft at any desired time point

$R P M=\frac{(\text { Pulse Frequency }, \text { pulses } / \text { sec }) \times(60 \text { sec } / \text { min })}{(\text { Sensor pulses } / \text { revolution })}$

where the tach ratio is the ratio of pulse/tach pulse. The instantaneous shaft speed over a 120 second acquisition period is seen in Figure 9. Wind speed, torque ripple, tower shadow, and measurement noise all contribute the oscillations seen in Figure 9.

The raw vibration signal has a DC offset as seen in Figure 10. While most accelerometers are AC-coupled, the ones used to monitor this wind turbine were DC-coupled. The DC type accelerometers are useful for measuring low frequency signals with both constant or dynamic accelerations (White, Adams,

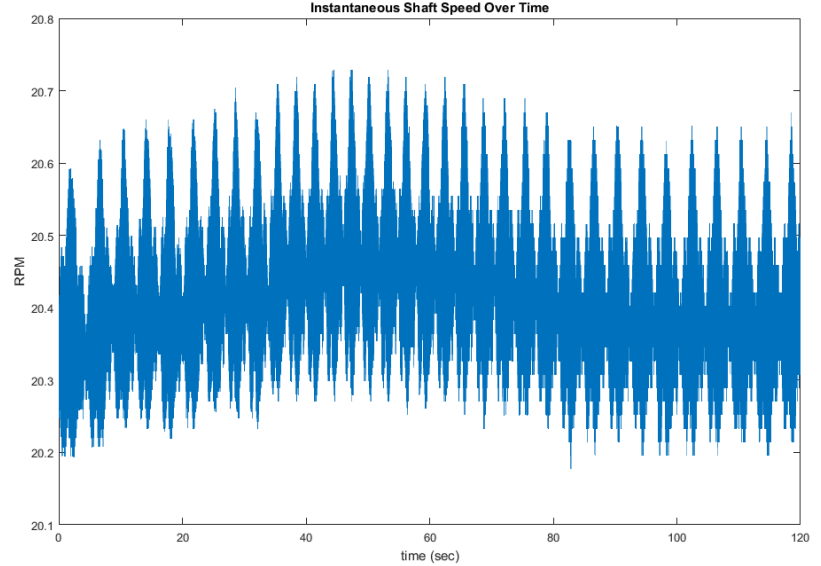

Figure 9. Calculated instantaneous shaft speed over the 120 second acquisition period.

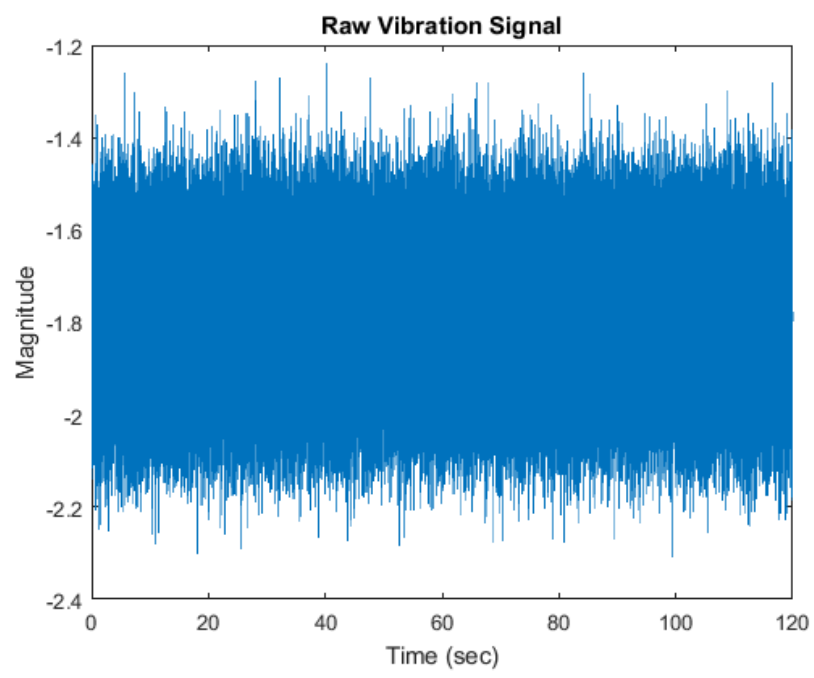

Figure 10. Typical raw vibration signal from main bearing

\& Rumsey, 2009). The signals of interest range between 0.15 and $5 \mathrm{~Hz}$. The DC type accelerometer can be used for signals 0 and $100 \mathrm{~Hz}$ (White et al., 2009). This DC offset is removed before performing the angle domain FFT. If the DC offset were present in the signal for the FFT, the largest spectral line in the frequency spectrum would be the zero frequency, which is not very informative or useful.

The vibration signal, with the DC offset removed, is then resampled according to the reference, tachometer signal. This new signal has constant angular spacing and can be seen in Figure 11. It's generally good practice to view the vibration data in the time domain before working with the data in another domain. Significant outliers and large trends can be seen in the time domain upon visual inspection. For a more detailed analysis, a Fourier transform needs to be performed to view the frequency spectrum. 


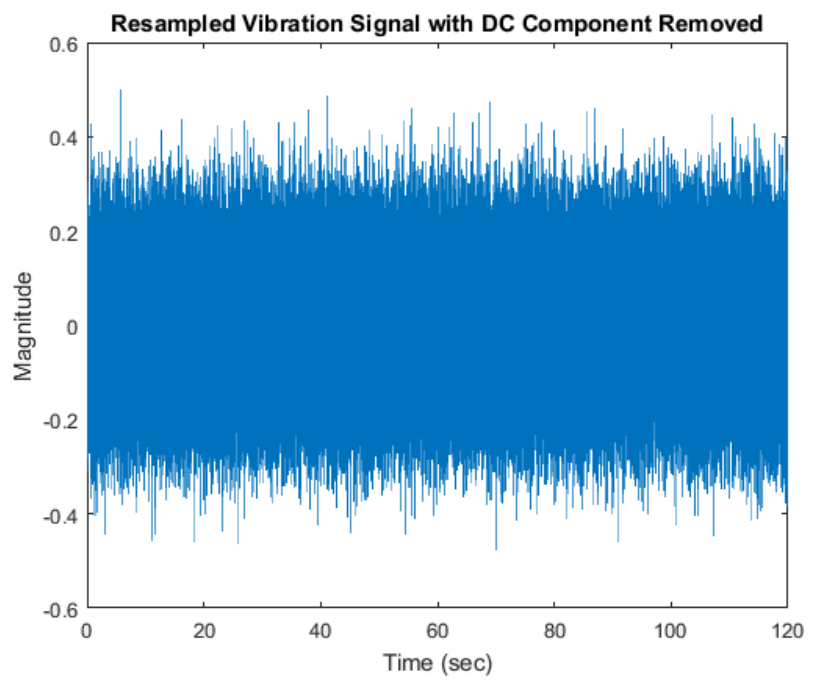

Figure 11. Resampled vibration signal from main bearing with DC component removed

\subsection{Order Analysis and Detection}

Although three machines were given in the original data set, the focus of these results is on machine 2 , because the data set shows clear signs of a bearing fault. The other machines' order spectrums will be shown as a comparison to see the differences between faulted and unfaulted signals. Figure 13 shows the full order spectrum from machine 2's main bearing during the first data acquisition period. The data are being sampled at a rate of 3052 samples/second, while the shaft is only turning at $0.33 \mathrm{~Hz}$. In an order spectrum, the shaft's turning speed is the order of one, and all other frequencies are multiples of the shaft frequency. The Nyquist criterion must still be satisfied, so the sampling rate must be at least twice that of the largest frequency. With the sampling rate being so much larger than the shaft frequency, an excessive number of orders can be seen. The bearing fault rates of $0.45,10.8,12.7$, and 15.3 cannot be seen in Figure 13, but other notable features from the system can. A large peak can be seen at order 90. The gearbox in a wind turbine commonly has a ratio of 90:1. The gearbox increases the rotational speed of the slow main-shaft to a higher speed, which more suitable for a generator. This signal is very loud as the gear's teeth push past one another. Harmonics of this signal can be seen at the order of 180,270, and 360. Another large peak can be seen around the order of 2,500. This peak is likely associated with a gear on a higher speed shaft further down the wind turbine drivetrain. It can also be seen that the main bearing signal has a large amount of noise over all frequency spectrum. This noise is large compared to the noise seen by the carrier bearing in Figure 20.

Machine 1, 2, and 3's order spectrums have been plotted to illustrate the similarities in the overall order spectrum seen in figures 12,13, and 14, respectively. Consistencies can be

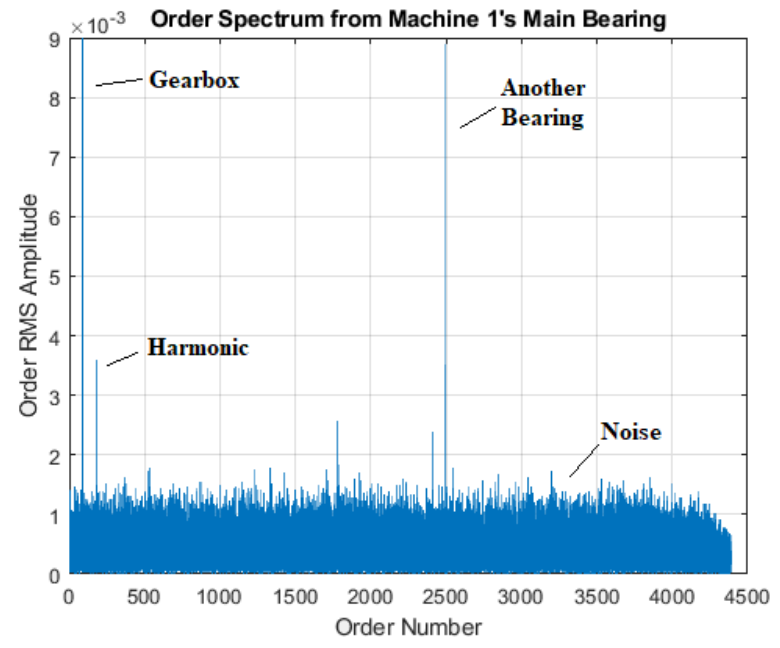

Figure 12. Complete order spectrum of Machine 1's main bearing from the first data acquisition period.

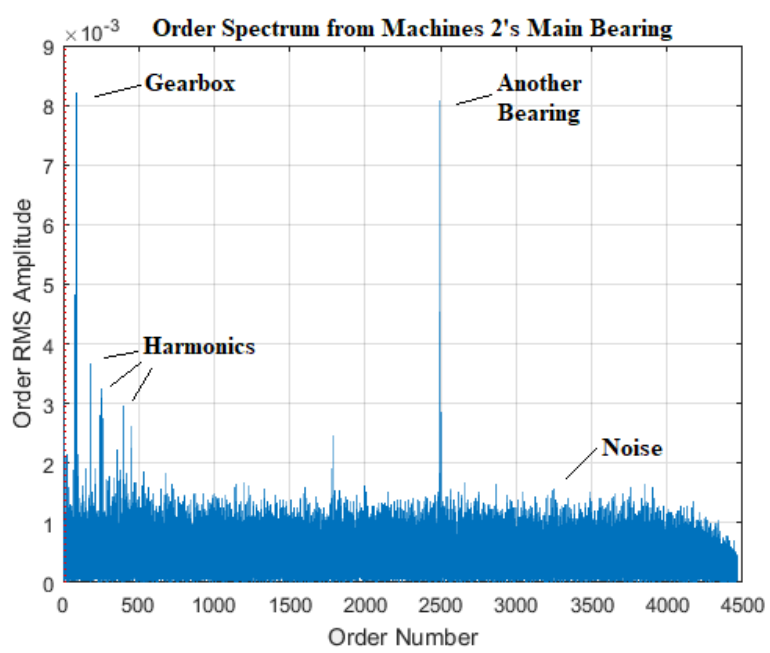

Figure 13. Complete order spectrum of Machine 2's main bearing from the first data acquisition period.

seen between the level of noise present and the other features, such as the gearbox and the higher frequency bearing.

Machine 2's data set was taken over nine time periods. For conciseness, emphasis is placed on the beginning, middle, and end of this data set, which correspond to acquisition 1, 5 , and 9 in the figure titles, respectively. Figure 15 shows the same data as Figure 13, but is refocused to display the first twenty orders. It is easy to see the bearing fault rates at this scale. The fault rates and shaft frequency are marked with a dashed red line.

The two largest peaks seen in Figure 15 are the order of 1 and 12.7. These orders correspond to the shaft frequency and the outer race fault frequency. The shaft order has an RMS amplitude of 0.0033 , while the outer race fault order has an 


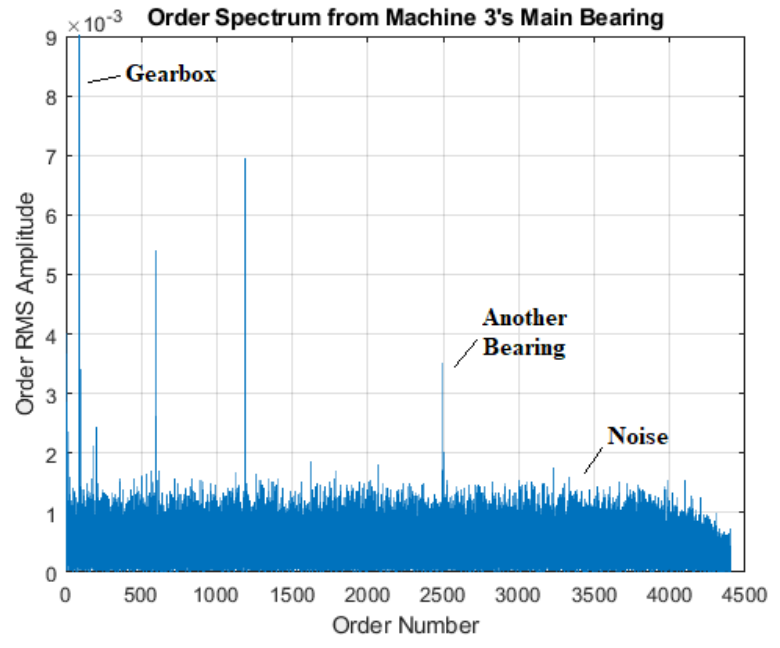

Figure 14. Complete order spectrum of Machine 3's main bearing from the first data acquisition period.

RMS amplitude of 0.0021 . Both amplitudes are well above the noise amplitude of 0.001 .

The zoomed-in order spectrums for machine 1 and 3 can be seen in figures 16 and 17, respectively. The shaft and fault frequencies have been marked with dashed lines for easy recognition. Machine 1 shows no noticeable fault frequencies with the only peak of note being the shaft frequency located at order 1. Machine 3, in figure 17, has a busier order spectrum. Significant peaks can be seen at order 1, 3, and 6. The first order is the shaft frequency. The third and sixth order are most likely related to the number of wind turbine blades and potentially a harmonic. No major peaks can be seen at any of the bearing fault frequencies leading to the conclusion that both machine 1 and 3's main bearings are currently healthy.

Figure 18 is the order spectrum of the main bearing from the fifth acquisition period of machine 2 . This is the midpoint of the data set. The peaks located at shaft and outer race fault orders are still present. The shaft order peak remained constant with an RMS amplitude of 0.0033 , while the outer race fault order increased slightly to an RMS amplitude of 0.0025. A new peak has developed at the order of 3, which could potentially be related to the wind turbine blades. Most wind turbines have three blades, but more information about this system would need to be known before the new peak can be confidently identified as relating to the blades. The new peak is the largest in this window with an RMS amplitude of almost 0.0035 . A harmonic of this peak can be seen at the order of 6 .

Figure 19 shows the order spectrum from machine 2's main bearing during the final acquisition period. The system either fails or undergoes maintenance after this acquisition period. The two major peaks in Figure 19 are once again the shaft and outer race fault orders. The peak relating to the wind tur-

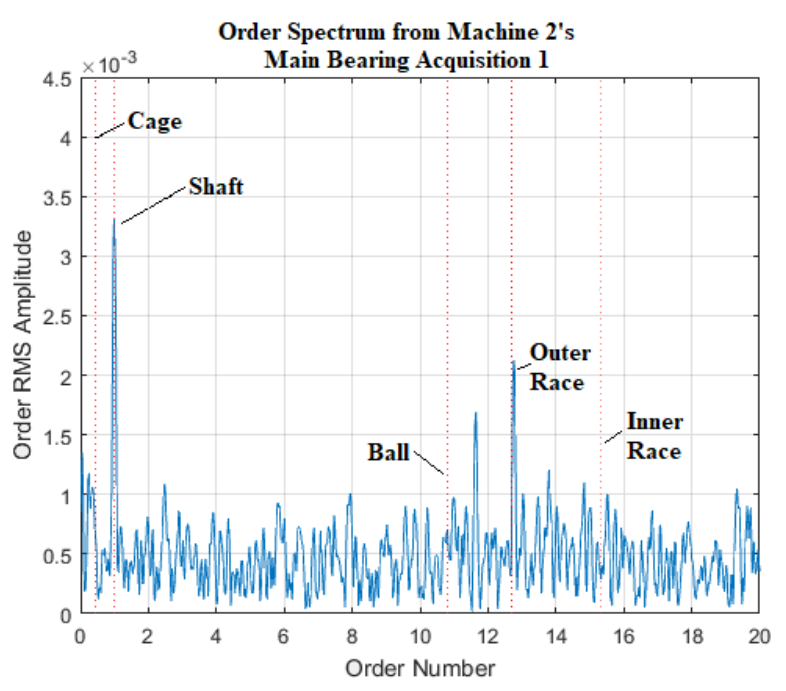

Figure 15. Order spectrum of Machine 2's main bearing from the first data acquisition period showing only first 20 orders. Bearing characteristic fault rates and the shaft order are indicated with dashed red lines. The shaft is located at order = 1. The cage, ball, outer race, and inner race correspond to the orders of $0.45,10.8,12.7$, and 15.3 , respectively.

bine blades has settled down to an RMS amplitude of 0.0018 , while the shaft and outer race fault RMS amplitudes are at an all time high of 0.0039 and 0.0033 , respectively. The value of $0.0039 \mathrm{~g}$ 's may not seem like a lot, but for such a low frequency shaft $(0.33 \mathrm{~Hz})$, it corresponds to a velocity of 0.78 in/sec RMS and a displacement of $25.8 \mathrm{~mm}$ peak-to-peak. There is also a "skirt" starting to develop in the spectrum around the outer race fault frequency in 19. The skirt refers to the frequencies immediately adjacent to the bearing fault frequency can still be related to the fault itself. Bearings are quasi-stationary, which means that they slip in relation to the shaft turning. Even with perfect knowledge of the geometry, the characteristic fault frequencies are not exact (Bechhoefer et al., 2013).

With the early presence of the outer race fault order in Figure 15 , it can be concluded the outer race defect was introduced early in the bearing's life. This could be due to a variety of reasons including: manufacturing error, electrical pitting or shock loading during installation (Tandon \& Choudhury, 1999).

The same analysis was completed for Machine 2's carrier bearing. The carrier bearing has the same dimensions as the main bearing and is located on the same shaft. The carrier bearing is located between the main bearing and the gearbox. Because of its dimensions, the carrier bearing and the main bearing have the same fault frequencies. The full order spectrum of machine 2's carrier bearing during the first data acquisition period can be seen in Figure 20. The noise level 


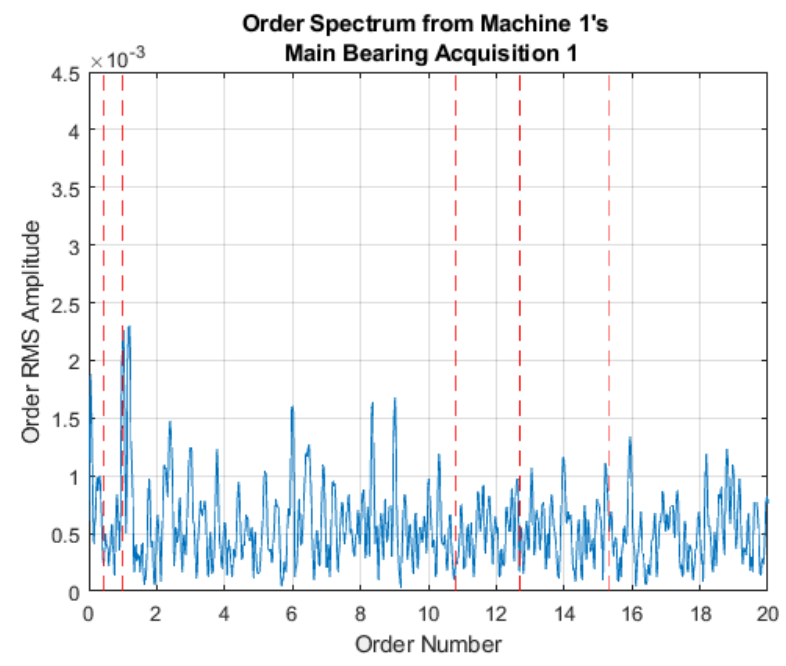

Figure 16. Order spectrum of Machine 1's main bearing from the first data acquisition period showing only first 20 orders. Key orders are indicated with red dashed lines: $f_{s}=1 ; f_{f t}=$ $0.45 ; f_{b s}=10.8 ; f_{\text {or }}=12.7 ; f_{\text {ir }}=15.3$.

across all frequencies is much lower than that of the main bearing. The largest amplitude in this spectrum is related to the gearbox at the order of 90. The fault frequency signal may be small, but it is still at least twice the amplitude of the surrounding noise in the other frequency bins.

Figures 23, 24, and 25 in the appendix show the order spectrum of machine 2's carrier bearing over the first, fifth, and last acquisition period, respectively. These plots show the same bearing fault with a similar fault progression as the main bearing. However, the carrier bearing's RMS amplitudes are an order of magnitude smaller than that of the main bearing for both the shaft and outer race fault peaks. Without further knowledge of the location of the sensors, it is difficult to determine whether the outer race fault peak seen in the main bearing's order spectrum is related to the carrier bearing's outer race fault peak. It is hard to imagine a scenario where both bearings experience the same fault and the damage progresses in such a similar fashion. With the RMS amplitude of the main bearing's fault rate being an order of magnitude larger than that of the carrier bearing's, it appears that the fault resides in the main bearing, but is still being picked up by the carrier bearing's accelerometer due to their close proximity.

\subsection{Prognostic Parameter Identification}

Since the fault has been diagnosed, finding an appropriate prognostic parameter to estimate the bearing's remaining useful life becomes the next priority. These parameters are typically established from expert judgment, but are compared objectively with other potential prognostic parameters through their monotonicity, trendability, and prognosability (Coble \& Hines, 2012). Trendability and prognosability are both features of a population of prognostic parameters. This fault

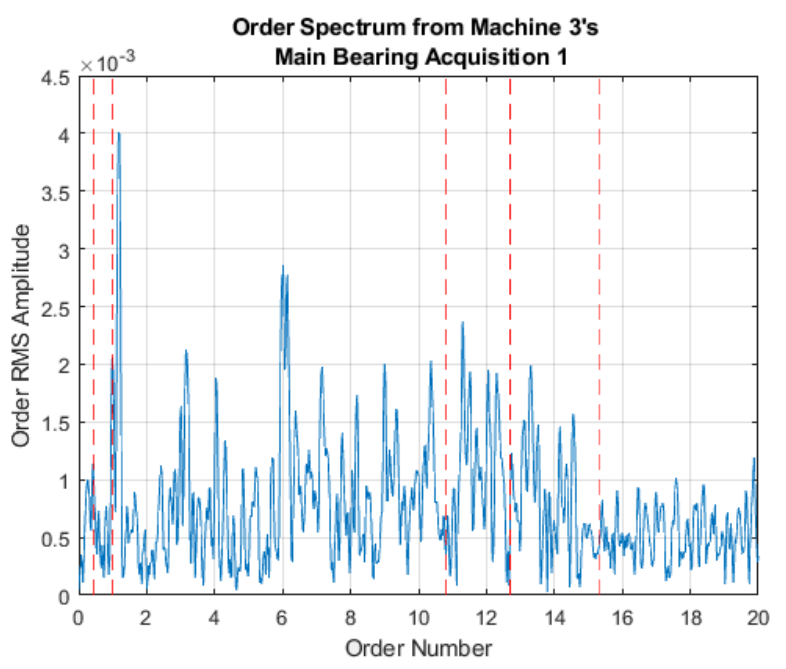

Figure 17. Order spectrum of Machine 3's main bearing from the first data acquisition period showing only first 20 orders. Key orders are indicated with red dashed lines: $f_{s}=1 ; f_{f t}=$ $0.45 ; f_{\text {bs }}=10.8 ; f_{\text {or }}=12.7 ; f_{\text {ir }}=15.3$

only appeared in one of the data sets, so these two features were not evaluated. Monotonicity describes the positive or negative tendency of the data. Monotonicity is based off the assumption that the fault can only grow, because the system cannot self-heal (Coble \& Hines, 2012). The monotonicity of the signal was used to evaluate potential prognostic parameter candidates.

The two prognostic parameters tested are peak RMS amplitude at the bearing fault frequency and total energy surrounding the bearing fault frequency. Peak RMS amplitude at the bearing rate was chosen as a candidate due to its simplicity. Upon first inspection of the data in the order spectrum, a visual peak at the bearing fault frequency may suggest that a fault of that type has occurred. Total energy surrounding the bearing fault frequency is slightly more sophisticated. Knowing that the bearing is quasi-stationary, all frequencies in the immediate area of the bearing fault could have been produced by the bearing fault itself. The slippage of the bearing is hypothesized to be the reason for the skirt that develops around the bearing fault order.

The peak RMS amplitudes of the bearing fault rate for the main bearing are plotted in Figure 21. There are nine total acquisition periods, so there are nine instances of the peak amplitude. There is no clear positive or negative trend seen in Figure 21 by this parameter over the life of the bearing. The peak RMS amplitude is therefore rejected as a potential prognostic parameter.

The other candidate as a prognostic parameter is the total energy around the outer race fault order. The total energy for this parameter was calculated by integrating from order 12.2 to 13.2. This effectively allows the bearing fault to slip for- 


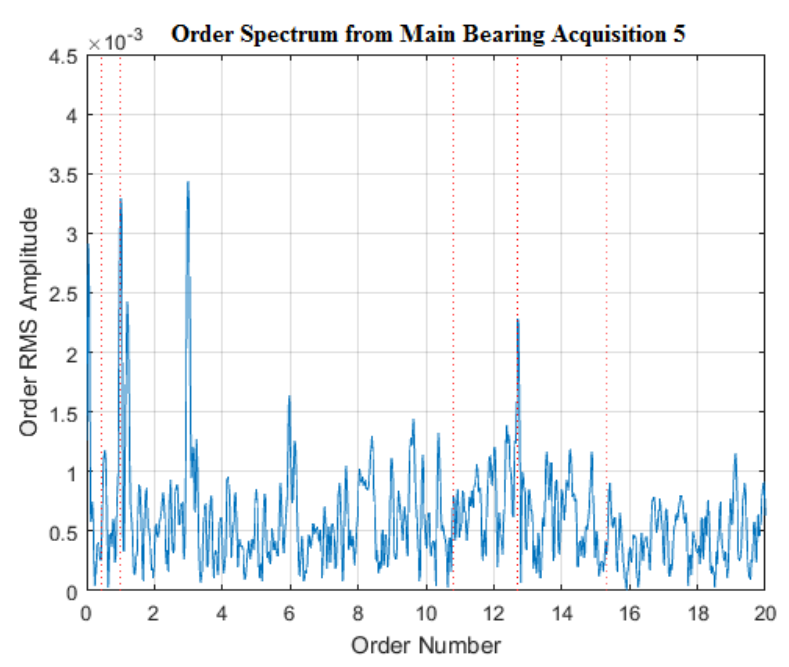

Figure 18. Order spectrum of Machine 2's main bearing from the fifth data acquisition period showing only the first 20 orders. Key orders are indicated with red dashed lines: $f_{s}=1 ; f_{f t}=0.45 ; f_{b s}=10.8 ; f_{\text {or }}=12.7 ; f_{\text {ir }}=15.3$

ward or backward equal to one half of the shaft frequency and still contribute to the prognostic parameter. This parameter was selected on a priori knowledge that the rolling elements inside bearings can slip. Energy surrounding the fault's order may be related to the fault itself. The width of the interval around the fault's order was chosen to accommodate as much surrounding orders as possible without overlapping orders of interest, such as the cage order of 0.45 and the shaft order of 1. The energy surrounding the fault's order in the main bearing in plotted in Figure 22. The overall trend of this plot is positive with a negative dip during the third and eighth acquisition. The majority of the data passes the monotonicity criteria, so this variable could be a potential prognostic parameter.

The energy surrounding the outer race fault frequency was plotted for both machines 1 and 3 in figures 26 and 27, respectively. Both of these bearings were deemed healthy, so no major trends are expected in these figures. There is no clear monotonic trend in either of these signals, but the scale seems to be similar to that of the faulted signal seem in machine 2 . The peak RMS value for the outer race fault frequency for both machine 1 and 3 can be seen in figures 28 and 29, respectively. These figures show a clear difference from the magnitude seen in the faulted signal and that of the unfaulted signals. These magnitudes are significantly smaller and are within the range of the surrounding noise. It may be useful to combine the results from the total energy and peak RMS values; since the total energy exhibits a useful monotonic trend, while the peak RMS value is the major determining factor for fault diagnosis.

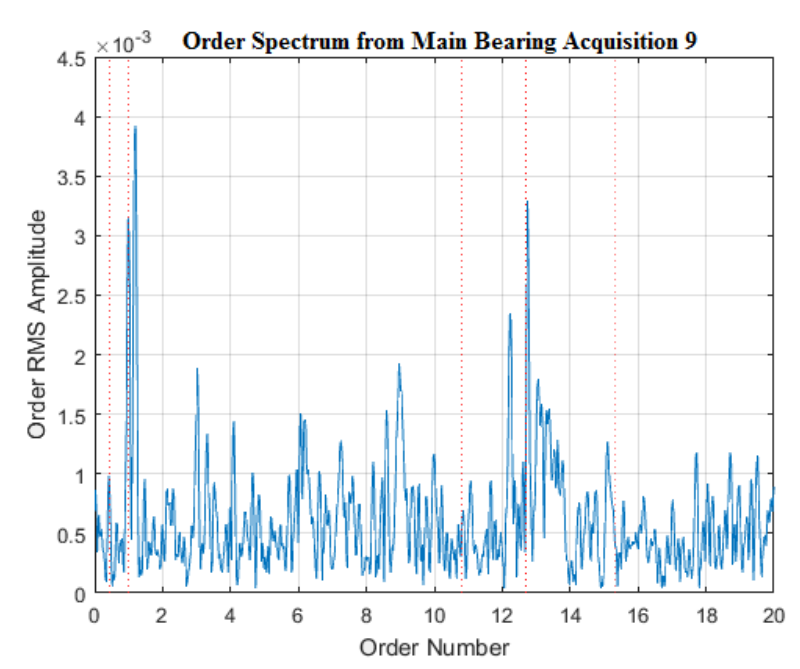

Figure 19. Order spectrum of Machine 2's main bearing from the last data acquisition period showing only the first 20 orders. Key orders are indicated with red dashed lines: $f_{s}=1 ; f_{f t}=0.45 ; f_{b s}=10.8 ; f_{\text {or }}=12.7 ; f_{\text {ir }}=15.3$

\section{Conclusions}

Wind turbines shafts have nonstationary frequencies that cannot be treated with traditional stationary frequency analysis techniques. With adaptive resampling, signals that are not stationary in the time domain can resampled to appear stationary in the angle domain. An angle domain FFT can then be applied to the newly resampled signal. Order tracking allows the faults to be distinguished easily. These techniques were implemented on vibration data collected at the main and carrier bearings of a wind turbine. An outer race fault was detected for machine 2 . This fault was confirmed in postmortem examinations. The damage propagation from this fault was slow due to the low shaft speed. A higher RPM shaft sees more passes from the rolling elements over a given period of time. This would lead to more impacts, faster damage accumulation, and a shorter bearing life. Once a fault is detected for a bearing on the wind turbine's main shaft, there should be plenty of time to monitor the fault and schedule appropriate maintenance actions during a routine outage.

\section{ACKNOWLEDgMent}

The research presented here is supported by the Nuclear Energy University Program of the U.S. Department of Energy. The authors gratefully acknowledge Dr. Eric Bechhoefer and GPMS for the wind turbine bearing data analyzed in this work.

\section{REFERENCES}

Bechhoefer, E., Hecke, B. V., \& He, D. (2013). Processing for improved spectral analysis [Journal Article]. phmsoci- 


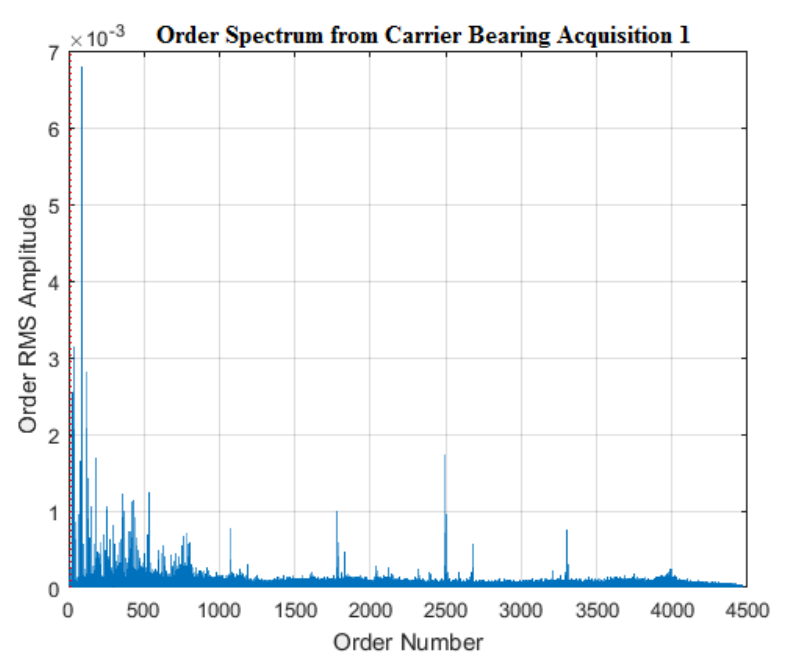

Figure 20. Complete order spectrum of Machine 2's carrier bearing from the first data acquisition period.

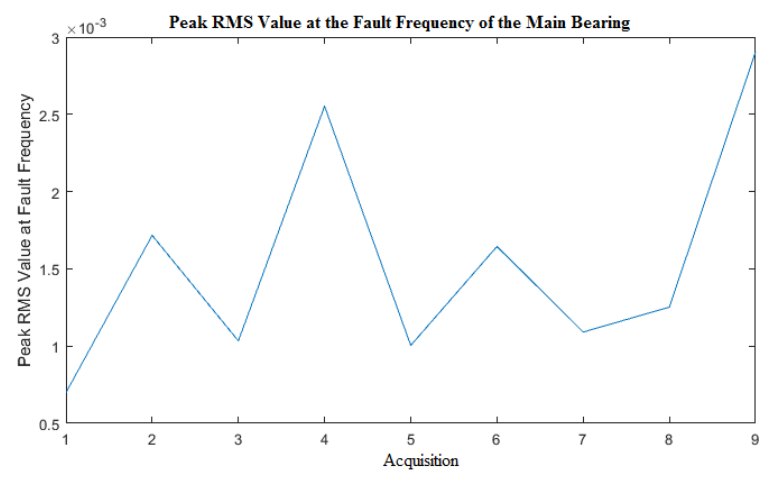

Figure 21. Peak RMS value of the fault frequency for the main bearing of Machine 2 for each data acquisition period.

ety, 4. Retrieved from http: / / www . phmsociety . org/node/1001/

Bechhoefer, E., \& Kingsley, M. (2009). A review of time synchronous average algorithms [Conference Paper]. San Diego, CA: Annual conference of the Prognostics and Health Management society.

Blough, J. R. (2006). Adaptive resampling - transforming from the time to the angle domain [Journal Article]. Proceedings of International Modal Analysis Conference(24), 315-329.

Coble, J., \& Hines, J. W. (2012). Identifying suitable degradation parameters for individual-based prognostics [Book Section]. In S. Kadry (Ed.), Prognostics of engineering systems: Methods and techniques (p. 135150).

Colombo, J., Arora, R., Depace, N. L., \& Vinik, A. I. (2015). Clinical autonomic dysfunction: Measurement, indications, therapies, and outcomes [Book]. Switzerland: Springer.

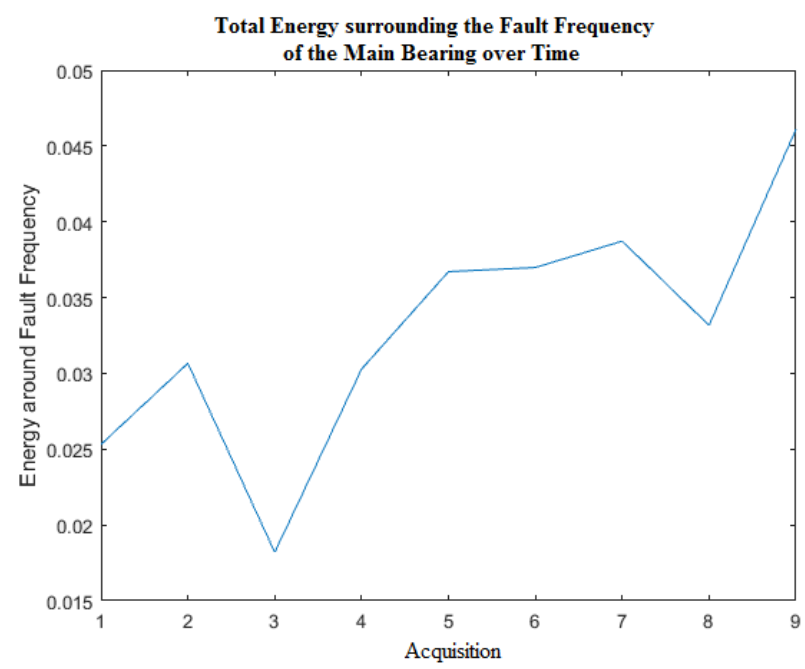

Figure 22. Total energy around the fault frequency of the main bearing of Machine 2 for each data acquisition period.

Dolan, D. S. L., \& Lehn, P. W. (2006, Sept). Simulation model of wind turbine $3 p$ torque oscillations due to wind shear and tower shadow. IEEE Transactions on Energy Conversion, 21(3), 717-724. doi: 10.1109/ TEC.2006.874211

Feldman, M. (2011). Hilbert transform applications in mechanical vibration. John Wiley and Sons, Ltd.

Holtz, J., \& Springob, L. (1996, Apr). Identification and compensation of torque ripple in high-precision permanent magnet motor drives. IEEE Transactions on Industrial Electronics, 43(2), 309-320. doi: 10.1109/41.491355

Huang, N., \& Shen, S. (2014). Hilbert-huang transform and its applications 2nd edition [Book]. Singapore: World Scientific Publishing Co.

Hussain, S., \& Gabbar, H. A. (2013). Vibration analysis and time series prediction for wind turbine gearbox prognostics. IJPHM Special Issue on Wind Turbine PHM (Color), 69.

Jardine, A. K. S., Lin, D., \& Banjevic, D. (2006). A review on machinery diagnostics and prognostics implementing condition-based maintenance [Journal Article]. Mechanical Systems and Signal Processing, 20, 1483-1510.

Li, R., \& Frogley, M. (2013). On-line fault detection in wind turbine transmission system using adaptive filter and robust statistical features. IJPHM Special Issue on Wind Turbine PHM (Color), 115.

Niknam, S. A., Thomas, T., Hines, J. W., \& Sawhney, R. (2013). Analysis of acoustic emission data for bearings subject to unbalance. International Journal of Prognostics and Health Management, 4, 80-89.

Rai, V. K., \& Mohanty, A. R. (2006). Bearing fault diagnosis using fft of intrinsic mode functions in hilberthuang transform [Journal Article]. ScienceDirect, Mechani- 
cal Systems and Signal Processing(21), 2607-2615.

Tandon, N., \& Choudhury, A. (1999). A review of vibration and acoustic measurement methods for the detection of defects in rolling element bearings [Journal Article]. Tribology International, 32(8), 469-480. Retrieved from http:// wWw.sciencedirect.com/science/ article/pii/s0301679X99000778 https://doi.org/10.1016/S0301-679X(99)00077-8

Tolstov, G. P. (1962). Fourier series, translated by richard $a$. silverman [Book]. New York: Dover Publications.

White, J. R., Adams, D. E., \& Rumsey, M. A. (2009). Operational load estimation of a smart wind turbine rotor blade. In Health monitoring of structural and biological systems 2009 (Vol. 7295, p. 72952D).

Williams, T., Ribadeneira, X., Billington, S., \& Kurfess, T. (2001). Rolling element bearing diagnostics in run-tofailure lifetime testing [Journal Article]. Mechanical Systems and Signal Processing, 15, 979-993.

WWEA. (2012). Quarterly bulletin. World Wind Energy Association Bulletin, 3, 30-36.

Zhang, H.-G., Zhang, S., \& Yin, Y.-X. (2014). A novel improved elm algorithm for a real industrial application [Journal Article]. Mathematical Problems in Engineering, 2014, 1-7. doi: 10.1155/2014/824765

Zhu, J., Yoon, J. M., He, D., Qu, Y., \& Bechhoefer, E. (2013). Lubrication oil condition monitoring and remaining useful life prediction with particle filtering. International Journal of Prognostics and Health Management, 4, 124-138.

Zipp, K. (2012). Understanding cost for large wind-turbine drivetrains [Web Page]. Retrieved from http:// www . windpowerengineering.com/design/ mechanical/understanding-costs-for -large-wind-turbine-drivetrains/

\section{BIOGRAPHIES}

Cody M. Walker received his B.S. degree in Nuclear Engineering from the University of Tennessee in 2015. After completing his B.S., he received a 3-year US Department of Energy Office of Nuclear Energy Integrated University Program Graduate Fellowship to pursue his graduate education. He completed his M.S. in Nuclear Engineering in 2017. He is currently working toward his Ph.D. in Nuclear Engineering focusing on fault detection, diagnostics, and prognostics of rotating equipment operating under non-stationary conditions. He is a member of the American Nuclear Society.

Jamie B. Coble received her B.S. degree in Nuclear Engineering and Mathematics from the University of Tennessee in 2005. She completed her MS in Nuclear Engineering in 2006, MS in Reliability and Maintenance Engineering in 2009, and $\mathrm{PhD}$ in Nuclear Engineering in 2010, all at the University of Tennessee. She worked for two years at Pacific Northwest National Laboratory before returning to the University of Tennessee in 2013 as an Assistant Professor of Nuclear Engineering. Her research interests include process monitoring, equipment condition assessment, online risk assessment, and prognostics-informed control. She is a member of the American Nuclear Society, US Women in Nuclear, International Society of Automation, and International Society of Engineering Asset Management, and a senior member of the Institute of Electrical and Electronics Engineers.

\section{APPENDIX}

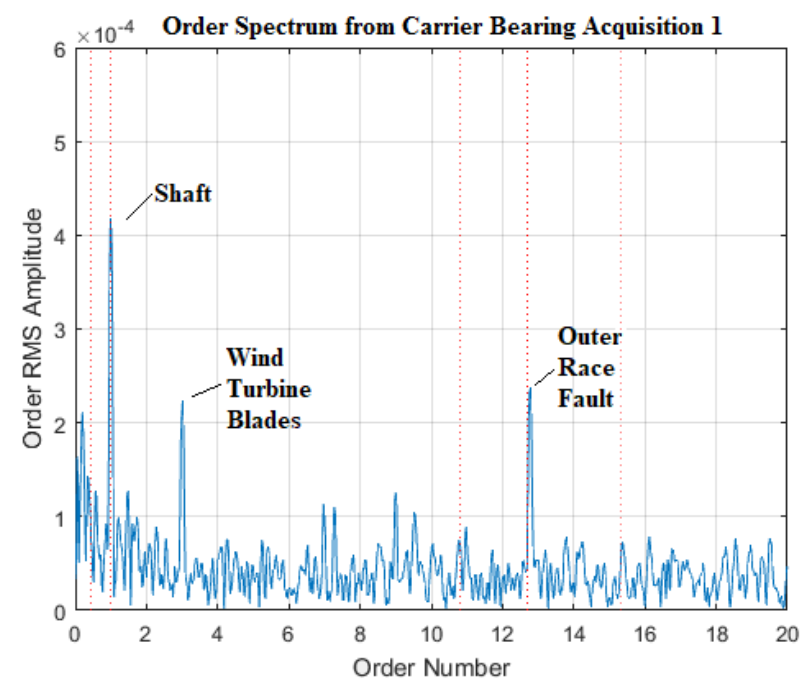

Figure 23. Order spectrum of Machine 2's carrier bearing from the first data acquisition period showing only the first 20 orders. Key orders are indicated with red dashed lines: $f_{s}=1 ; f_{f t}=0.45 ; f_{b s}=10.8 ; f_{\text {or }}=12.7 ; f_{\text {ir }}=15.3$ 


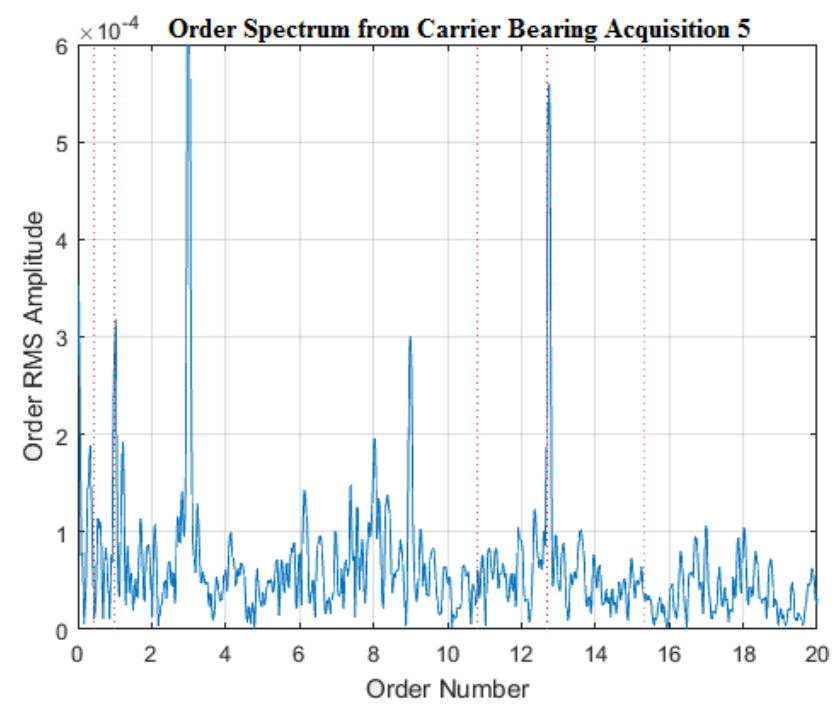

Figure 24. Order spectrum of Machine 2's carrier bearing from the fifth data acquisition period showing only the first 20 orders. Key orders are indicated with red dashed lines: $f_{s}=1 ; f_{f t}=0.45 ; f_{b s}=10.8 ; f_{\text {or }}=12.7 ; f_{\text {ir }}=15.3$

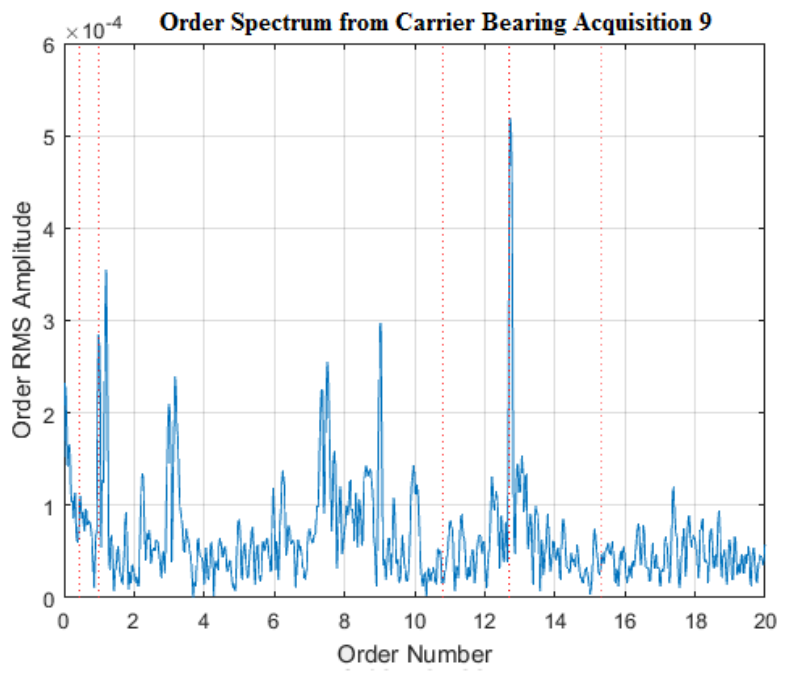

Figure 25. Order spectrum of Machine 2's carrier bearing from the last data acquisition period showing only the first 20 orders. Key orders are indicated with red dashed lines: $f_{s}=1 ; f_{f t}=0.45 ; f_{b s}=10.8 ; f_{\text {or }}=12.7 ; f_{i r}=15.3$

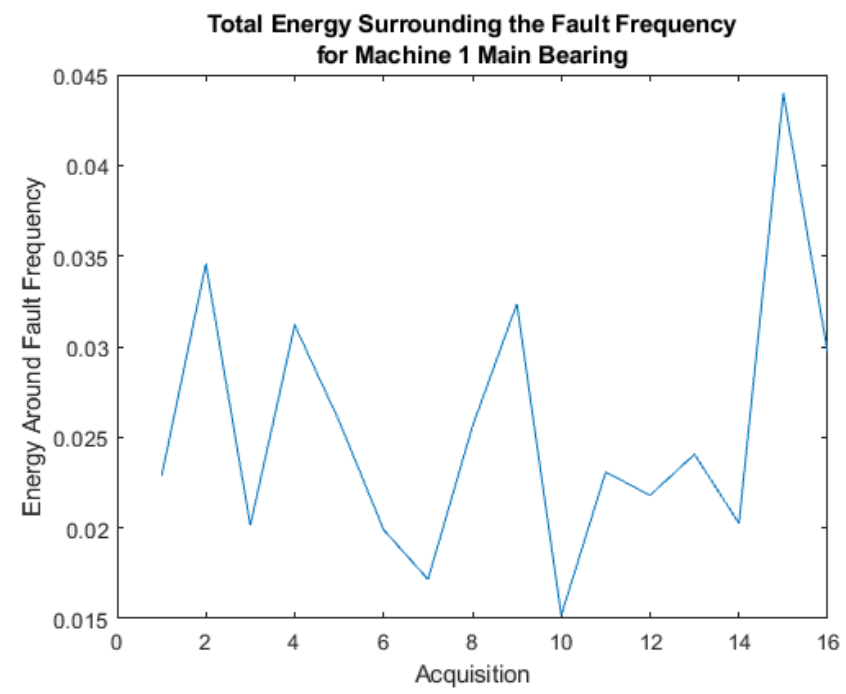

Figure 26. Total energy around the fault frequency of the main bearing of Machine 1 for each data acquisition period.

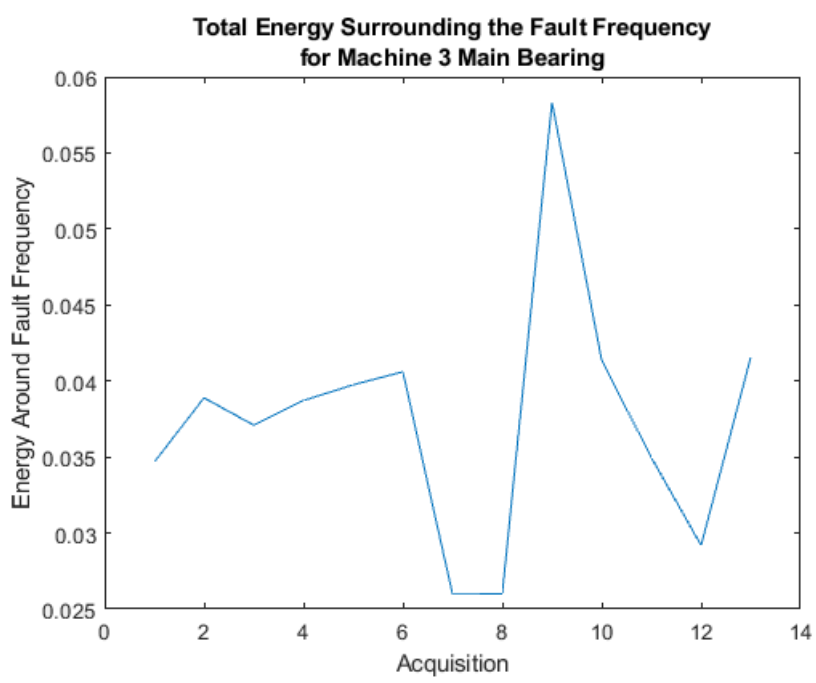

Figure 27. Total energy around the fault frequency of the main bearing of Machine 3 for each data acquisition period. 


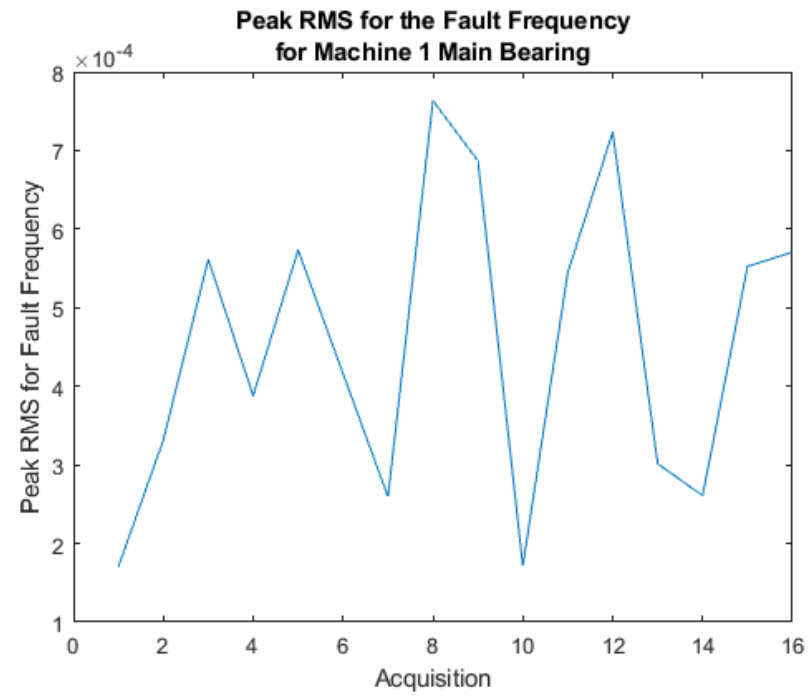

Figure 28. Peak RMS for the fault frequency of the main bearing of Machine 1 for each data acquisition period.

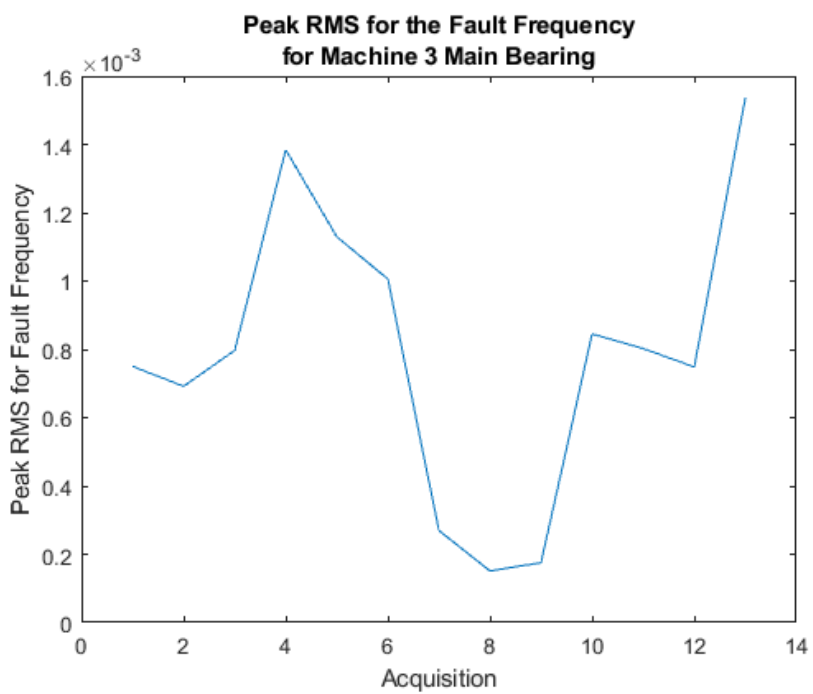

Figure 29. Peak RMS for the fault frequency of the main bearing of Machine 3 for each data acquisition period. 